JIRSS (2018)

Vol. 17, No. 1, pp 109-129

DOI: 10.29252/jirss.17.1.109

\title{
A New Method for Generating Continuous Bivariate Distribu- tion Families
}

\author{
Masoud Ganji ${ }^{1}$, Hossein Bevrani ${ }^{2}$ and Nasrin Hami Golzar ${ }^{3}$ \\ ${ }^{1,3}$ Department of Statistics, University of Mohaghegh Ardabili, Ardabil, Iran. \\ ${ }^{2}$ Department of Statistics, University of Tabriz, Tabriz, Iran.
}

Received: 26/11/2014, Revision received: 20/02/2017, Published online: 19/05/2018

\begin{abstract}
Recently, it has been observed that a new method for generating continuous distributions, $T-X$ family, can be quite effectively used to analyze the data in one dimension. The aim of this study is to generalize this method to two dimensional space so that the marginals would have $T-X$ distributions. So, several examples and properties of this family have been presented. As an application, a special distribution of this family, called bivariate Weibull-Rayleigh-Rayleigh, is fitted to a data set and is shown to have a better fit.
\end{abstract}

Keywords. Bivariate distribution, Shannon entropy, $T-X$ family.

MSC: 62E15; 60E05.

\section{Introduction}

Statistical distributions are commonly used for describing real world phenomena. Many generalized classes of univariate distributions have been upgraded to bivari-

Masoud Ganji (mganji@uma.ac.ir)

Corresponding Author: Hossein Bevrani (bevrani@tabrizu.ac.ir)

Nasrin Hami Golzar (nasrin.hami@uma.ac.ir) 
ate and multivariate cases and applied to describe various phenomena. Recently, several theoretical books on multivariate non-normal distributions have been published: Hutchinson and Lai (1990), Joe (1997), Arnold et al. (1999), Kotz et al. (2000) and Nelsen (2006). Finally, Alzaatreh et al. $(2013, a)$ presented a new method for generating univariate families of continuous distributions. The resulting family, $T-X$, has many new distributions as its members. Let $r(t)$ be the probability density function (p.d.f.) of a random variable $T \in[a, b],-\infty \leq a<b \leq+\infty$. Let $W(F(x))$ be a function of the cumulative distribution function (c.d.f.) $F(x)$ of any random variable $X$ so that $W(F(x))$ satisfies the following conditions:

1. $W(F(x)) \in[a, b]$

2. $W(F(x))$ is differentiable and monotonically non-decreasing.

3. $W(F(x)) \rightarrow a$ as $x \rightarrow-\infty$ and $W(F(x)) \rightarrow b$ as $x \rightarrow+\infty$.

A method for generating new families of distributions is presented in the following definition.

Definition 1.1. Let $X$ be a random variable with p.d.f. $f(x)$ and c.d.f. $F(x)$. Let $T$ be a continuous random variable with p.d.f. $r(t)$ defined on $[a, b]$. The c.d.f. of a new family of distributions is defined as

$$
G(x)=\int_{a}^{W(F(x))} r(t) d t
$$

where $W(F(x))$ satisfies the three conditions mentioned above. The c.d.f. $G(x)$ in (1.1) can be written as $G(x)=R(W(F(x)))$, where $R(t)$ is the c.d.f. of T. The corresponding p.d.f. associated with (1.1) is

$$
g(x)=\left(\frac{\partial}{\partial x} W(F(x))\right) r(W(F(x)))
$$

When the support of $T$ is $[a,+\infty), a \geq 0, W(F(x))$ can be defined as $-\log (1-F(x))$, $\frac{F(x)}{1-F(x)}$ and $-\log \left(1-F^{\alpha}(x)\right)$, where $\alpha>0$. When the support of $T$ is $(-\infty,+\infty), W(F(x))$ can be defined as $\log (-\log (1-F(x))), \log \frac{F(x)}{1-F(x)}$ and $\log \left(-\log \left(1-F^{\alpha}(x)\right)\right)$. This method has already been discussed in detail in Alzaatreh et al. $(2013, a)$. Various distributions can be derived through this method. The gamma-half normal distribution and the gammanormal distribution were introduced by Alzaatreh and Knight (2013) and Alzaatreh et al. (2014), respectively. Also the gamma-Pareto and Weibull-Pareto distributions were introduced by Alzaatreh et al. (2012) and Alzaatreh et al. $(2013, b)$, respectively. The gamma-uniform and the logistic-uniform distributions were introduced by Torabi 
and Montazeri (2012) and Torabi and Montazeri (2014), respectively. In this paper, a new method is proposed to generate bivariate families of continuous distributions using the $T-X$ family named $(U, V)-X-Y$ distribution family.

\section{New Results and Generalizations}

Using the method of generating new distributions, $T-X$ family subsumes many wellknown distributions along with a vast array of new bivariate distributions. This section will generalize the method of $T-X$ distribution family to derive families of bivariate distributions by using two other univariate distributions as generators.

Let $r(u, v)$ be the p.d.f. of random vector $(U, V)$, where $U \in\left[a_{1}, b_{1}\right], V \in\left[a_{2}, b_{2}\right]$, $-\infty \leq a_{1}<b_{1} \leq+\infty,-\infty \leq a_{2}<b_{2} \leq+\infty$. Also let $W_{1}\left(F_{1}(x)\right)$ and $W_{2}\left(F_{2}(y)\right)$ be two functions of the c.d.f. $F_{1}(x)$ and c.d.f. $F_{2}(y)$ of random variables $X$ and $Y$, respectively, which satisfy the following conditions:

1. $W_{1}\left(F_{1}(x)\right) \in\left[a_{1}, b_{1}\right]$ and $W_{2}\left(F_{2}(y)\right) \in\left[a_{2}, b_{2}\right]$

2. $W_{1}\left(F_{1}(x)\right)$ and $W_{2}\left(F_{2}(y)\right)$ are differentiable and not decreasing

3. $\lim _{x \rightarrow-\infty} W_{1}\left(F_{1}(x)\right)=a_{1}, \lim _{x \rightarrow+\infty} W_{1}\left(F_{1}(x)\right)=b_{1}$

4. $\lim _{y \rightarrow-\infty} W_{2}\left(F_{2}(y)\right)=a_{2}, \lim _{y \rightarrow+\infty} W_{2}\left(F_{2}(y)\right)=b_{2}$.

Definition 2.1. Let $X$ be a random variable with p.d.f. $f_{1}(x)$ and c.d.f. $F_{1}(x)$ and $Y$ be another random variable with p.d.f. $f_{2}(y)$ and c.d.f. $F_{2}(y)$. Let $(U, V)$ be the continuous bivariate random vector with p.d.f. $r(u, v)$ and c.d.f. $R(u, v)$ defined on $\left[a_{1}, b_{1}\right] \times\left[a_{2}, b_{2}\right]$. The c.d.f. of $a$ new bivariate family of distributions is defined as

$$
G(x, y)=\int_{a_{1}}^{W_{1}\left(F_{1}(x)\right)} \int_{a_{2}}^{W_{2}\left(F_{2}(y)\right)} r(u, v) d v d u .
$$

which can be written as

$$
G(x, y)=R\left(W_{1}\left(F_{1}(x)\right), W_{2}\left(F_{2}(y)\right)\right) .
$$

The corresponding p.d.f. associated with (2.1) is

$$
g(x, y)=\left(\frac{\partial}{\partial x} W_{1}\left(F_{1}(x)\right)\right)\left(\frac{\partial}{\partial y} W_{2}\left(F_{2}(y)\right)\right) r\left(W_{1}\left(F_{1}(x)\right), W_{2}\left(F_{2}(y)\right)\right) .
$$


The c.d.f. in (2.1) is a composite function of $W_{1}, W_{2}, F_{1}, F_{2}$ and $r$. It is easy to show that (2.1) and (2.2) satisfy the conditions of c.d.f. and p.d.f., respectively. With regard the range of $(U, V)$, different forms of $W_{1}\left(F_{1}(x)\right)$ and $W_{2}\left(F_{2}(y)\right)$ can be obtained. In this article, when $(U, V)$ has the support $\mathfrak{R}^{2}$, we will focus on the forms $W(F(x))=\log \frac{F(x)}{1-F(x)}$ and $W(F(x))=\log (-\log (1-F(x)))$. When $(U, V)$ has the support $\mathfrak{R}_{+}^{2}$, we will focus on the forms $W(F(x))=\frac{F(x)}{1-F(x)}$ and $W(F(x))=-\log (1-F(x))$. The bivariate normal and bivariate logistic distributions are examples with support $\mathfrak{R}^{2}$. Moreover, the bivariate Weibull, bivariate gamma and bivariate Gumbel distributions have support $\mathfrak{R}_{+}^{2}$, (Kotz et al. (2000)). In the following, some properties of the $(U, V)-X-Y$ distribution family are presented.

Lemma 2.2. Let $(X, Y)$ be a bivariate random vector that follows the p.d.f. (2.2). Define $U=W_{1}\left(F_{1}(X)\right)$ and $V=W_{2}\left(F_{2}(Y)\right)$. Then, the random vector $(U, V)$ has p.d.f. $r(u, v)$.

Proof. The proof is straightforward, hence omitted.

The following theorem provides the marginals of the $(U, V)-X-Y$ distributions.

Theorem 2.3. Let $(X, Y)$ follow the p.d.f. (2.2). Then the marginals are

$$
g_{X}(x)=\left(\frac{\partial}{\partial x} W_{1}\left(F_{1}(x)\right)\right) r_{U}\left(W_{1}\left(F_{1}(x)\right)\right)
$$

and

$$
g_{Y}(y)=\left(\frac{\partial}{\partial y} W_{2}\left(F_{2}(y)\right)\right) r_{V}\left(W_{2}\left(F_{2}(y)\right)\right)
$$

In addition,

$$
E_{X, Y}(X)=E_{U}\left(F_{1}^{-1}\left(W_{1}^{-1}(U)\right)\right)
$$

and

$$
E_{X, Y}(Y)=E_{V}\left(F_{2}^{-1}\left(W_{2}^{-1}(V)\right)\right)
$$

where $U$ and $V$ follow the marginals of the p.d.f. $r(U, V)\left(r_{U}\right.$ and $\left.r_{V}\right)$.

Proof. Integrating (2.2) with respect to $x$ and $y$, respectively, the marginals $g_{X}(x)$ and 
$g_{Y}(y)$ will be obtained. In addition,

$$
\begin{aligned}
E_{X, Y}(X) & =\int_{X} \int_{Y} x\left(\frac{\partial}{\partial x} W_{1}\left(F_{1}(x)\right)\right)\left(\frac{\partial}{\partial y} W_{2}\left(F_{2}(y)\right)\right) r\left(W_{1}\left(F_{1}(x)\right), W_{2}\left(F_{2}(y)\right)\right) d y d x \\
& =\int_{X} x\left(\frac{\partial}{\partial x} W_{1}\left(F_{1}(x)\right)\right) \int_{Y}\left(\frac{\partial}{\partial y} W_{2}\left(F_{2}(y)\right)\right) r\left(W_{1}\left(F_{1}(x)\right), W_{2}\left(F_{2}(y)\right)\right) d y d x \\
& =\int_{X} x\left(\frac{\partial}{\partial x} W_{1}\left(F_{1}(x)\right)\right) r_{U}\left(W_{1}\left(F_{1}(x)\right)\right) d x \\
& =E_{U}\left(F_{1}^{-1}\left(W_{1}^{-1}(U)\right)\right) .
\end{aligned}
$$

$E_{X, Y}(Y)$ can be easily obtained too.

One can notice that the marginal densities are the same as the $T-X$ family of distributions which have already been introduced by Alzaatreh et al. $(2013, \mathrm{a})$. Some general models of the $(U, V)-X-Y$ distribution family will be studied in the following.

\section{Some General Models}

Let the support of the $r(u, v)$ be $\mathfrak{R}^{2}$, then various forms of $W_{1}\left(F_{1}(x)\right)$ and $W_{2}\left(F_{2}(y)\right)$ can be used in (2.2). We present some examples in the following.

Example 3.1. In (2.2), $W_{1}\left(F_{1}(x)\right)=\log \frac{F_{1}(x)}{1-F_{1}(x)}$ and $W_{2}\left(F_{2}(y)\right)=\log \frac{F_{2}(y)}{1-F_{2}(y)}$, then

$$
\begin{aligned}
g(x, y) & =\frac{f_{1}(x) f_{2}(y)}{F_{1}(x) F_{2}(y)\left(1-F_{1}(x)\right)\left(1-F_{2}(y)\right)} \\
& \times r\left(\log \frac{F_{1}(x)}{1-F_{1}(x)}, \log \frac{F_{2}(y)}{\left.1-F_{2}(y)\right)}\right) \\
& =\frac{h_{1}(x) h_{2}(y)}{F_{1}(x) F_{2}(y)} r\left(\log \frac{F_{1}(x)}{1-F_{1}(x)}, \log \frac{F_{2}(y)}{\left.1-F_{2}(y)\right)}\right),
\end{aligned}
$$

where $h_{1}(x)$ and $h_{2}(y)$ are the hazard functions of random variables $X$ with c.d.f. $F_{1}(x)$ and $Y$ with c.d.f. $F_{2}(y)$, respectively. The support of $g(x, y)$ depends on the supports of $F_{1}(x)$ and $F_{2}(y)$.

Define $F_{1}(x)=\frac{e^{x}}{1+e^{x}}$ and $F_{2}(y)=\frac{e^{y}}{1+e^{y}}$, then $g(x, y)=r(x, y)$. Hence the $(U, V)-X-Y$ family can be a generalization of the $(U, V)$ family. 
In Gray (1990), Gray has investigated and introduced some of the Shannon entropy properties in the univariate and multivariate cases. Also the reader can refer to Cover and Thomas (2006) and Shannon (1948).

Theorem 3.1. Given the bivariate random vector $(U, V)$ with p.d.f. $r(u, v)$ and the bivariate random vector $(X, Y)$ with p.d.f. (3.1), the Shannon entropy of $(X, Y)$ is

$$
\begin{aligned}
\eta_{X, Y} & =\eta_{U, V}+E_{U}(U)+E_{V}(V)+2 E\left(\log \left(1-\frac{e^{U}}{1+e^{U}}\right)\right) \\
& +2 E\left(\log \left(1-\frac{e^{V}}{1+e^{V}}\right)\right)-E_{U}\left(\log \left(f_{1}\left(F_{1}^{-1}\left(\frac{e^{U}}{1+e^{U}}\right)\right)\right)\right) \\
& -E_{V}\left(\log \left(f_{2}\left(F_{2}^{-1}\left(\frac{e^{V}}{1+e^{V}}\right)\right)\right)\right)
\end{aligned}
$$

where $\eta_{U, V}$ is the Shannon entropy of the bivariate random vector $(U, V)$.

Proof. According to the definition of Shannon entropy, $\eta_{X, Y}$ is

$$
\begin{aligned}
\eta_{X, Y} & =-E_{X, Y}(\log (g(X, Y))) \\
& =-E_{X, Y}\left(\log \left(f_{1}(X)\right)\right)-E_{X, Y}\left(\log \left(f_{2}(Y)\right)\right)+E_{X, Y}\left(\log \left(F_{1}(X)\right)\right) \\
& +E_{X, Y}\left(\log \left(F_{2}(Y)\right)\right)+E_{X, Y}\left(\log \left(1-F_{1}(X)\right)\right) \\
& +E_{X, Y}\left(\log \left(1-F_{2}(Y)\right)\right) \\
& -E_{X, Y}\left(\log r\left(\log \frac{F_{1}(X)}{1-F_{1}(X)}, \log \frac{F_{2}(Y)}{1-F_{2}(Y)}\right)\right) \\
& =-E_{X, Y}\left(\log \left(f_{1}(X)\right)\right)-E_{X, Y}\left(\log \left(f_{2}(Y)\right)\right) \\
& +E_{X, Y}\left(\log \frac{F_{1}(X)}{1-F_{1}(X)}\right)+E_{X, Y}\left(\log \frac{F_{2}(Y)}{1-F_{2}(Y)}\right) \\
& +2 E_{X, Y}\left(\log \left(1-F_{1}(X)\right)\right)+2 E_{X, Y}\left(\log \left(1-F_{2}(Y)\right)\right) \\
& -E_{X, Y}\left(\log r\left(\log \frac{F_{1}(X)}{1-F_{1}(X)}, \log \frac{F_{2}(Y)}{1-F_{2}(Y)}\right)\right)
\end{aligned}
$$

Define $U=\log \frac{F_{1}(X)}{1-F_{1}(X)}$ and $V=\log \frac{F_{2}(Y)}{1-F_{2}(Y)}$. Then

$$
-E_{X, Y}\left(\log r\left(\log \frac{F_{1}(X)}{1-F_{1}(X)}, \log \frac{F_{2}(Y)}{1-F_{2}(Y)}\right)\right)=-E_{U, V}(\log r(U, V))=\eta_{U, V}
$$

Also

$$
E_{X, Y}\left(\log \frac{F_{1}(X)}{1-F_{1}(X)}\right)=E_{U}(U)
$$


and

$$
E_{X, Y}\left(\log \frac{F_{2}(Y)}{1-F_{2}(Y)}\right)=E_{V}(V),
$$

where $E_{U}(U)$ and $E_{V}(V)$ are the expectations of random variables $U$ and $V$, respectively, whose density functions will be obtained from marginals of $r(u, v)$. Then the results are obtained.

Example 3.2. In (2.2), let define $W_{1}\left(F_{1}(x)\right)=\log \left(-\log \left(1-F_{1}(x)\right)\right)$ and $W_{2}\left(F_{2}(y)\right)=$ $\log \left(-\log \left(1-F_{2}(y)\right)\right)$. Then

$$
g(x, y)=\frac{f_{1}(x) f_{2}(y) r\left(\log \left(-\log \left(1-F_{1}(x)\right)\right), \log \left(-\log \left(1-F_{2}(y)\right)\right)\right)}{\left(1-F_{1}(x)\right)\left(1-F_{2}(y)\right) \log \left(1-F_{1}(x)\right) \log \left(1-F_{2}(y)\right)} .
$$

Define $F_{1}(x)=1-e^{-e^{x}}$ and $F_{2}(y)=1-e^{-e^{y}}$, then $g(x, y)=r(x, y)$. Then the $(U, V)-X-Y$ family can be a generalization of the $(U, V)$ family in this case, too.

Figure 1 indicates the relation between the $(U, V)-X-Y$ family and the $(U, V)$ family when the support of $r(u, v)$ is $\mathfrak{R}^{2}$.

Similar to Theorem 3.1, Shannon entropy of $(X, Y)$ for the p.d.f. given in Example 3.2 can be obtained as

$$
\begin{aligned}
\eta_{X, Y}= & \eta_{U, V}-E_{U}(U)-E_{V}(V)-E\left(e^{U}\right)-E\left(e^{V}\right)-E\left(\operatorname { l o g } f _ { 1 } \left(F_{1}^{-1}\left(1-e^{-e^{U}}\right)\right.\right. \\
& -E\left(\log f_{2}\left(F_{2}^{-1}\left(1-e^{-e^{V}}\right)\right)\right) .
\end{aligned}
$$

Some special models of the $(U, V)-X-Y$ distribution family will be studied in Section 4.

\section{Some Special Models}

\subsection{Bivariate Normal-X-Y Distribution Family}

Two random variables $X$ and $Y$ are said to have a bivariate normal distribution with parameters $\mu_{1}, \mu_{2}, \sigma_{1}, \sigma_{2}$ and $\rho$ if their joint p.d.f. is given by

$$
\begin{aligned}
f_{\text {Bnorm }}(u, v) & =\frac{1}{2 \pi \sigma_{1} \sigma_{2} \sqrt{1-\rho^{2}}} \exp \left(-\frac{1}{2\left(1-\rho^{2}\right)}\left(\left(\frac{u-\mu_{1}}{\sigma_{1}}\right)^{2}\right.\right. \\
& \left.\left.+\left(\frac{v-\mu_{2}}{\sigma_{2}}\right)^{2}-\frac{2 \rho\left(u-\mu_{1}\right)\left(v-\mu_{2}\right)}{\sigma_{1} \sigma_{2}}\right)\right)
\end{aligned}
$$


where $\mu_{1}, \mu_{2} \in \mathfrak{R}, \sigma_{1}, \sigma_{2} \in \mathfrak{R}_{+}, \rho \in(-1,1)$ and $u, v \in \mathfrak{R}$.

Using this p.d.f. and the $(U, V)-X-Y$ family method, the bivariate normal-X-Y distribution family can be introduced and examined. 


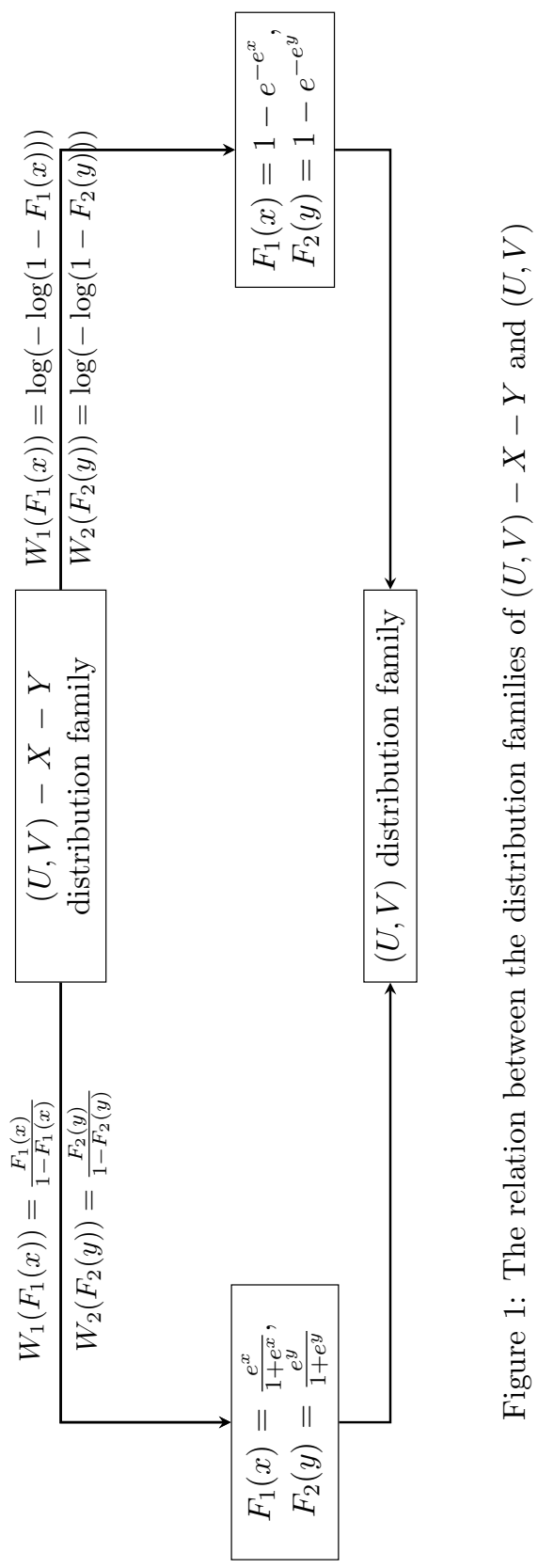


1. Let in (3.1) $r(x, y), F_{1}(x)$ and $F_{2}(y)$ be the bivariate normal p.d.f., the exponential c.d.f. with parameter $a$ and the exponential c.d.f. with parameter $b$, respectively. Then the bivariate normal-exponential-exponential type 1 (BNEE1) p.d.f. is obtained as

$$
\begin{aligned}
g(x, y)= & \frac{a b}{\left(1-e^{-a x}\right)\left(1-e^{-b y}\right)} f_{\text {Bnorm }}\left(\log \left(e^{a x}-1\right), \log \left(e^{b y}-1\right)\right) \\
= & \frac{a b}{2 \pi \sigma_{1} \sigma_{2} \sqrt{1-\rho^{2}}\left(1-e^{-a x}\right)\left(1-e^{-b y}\right)} \exp \left(-\frac{1}{2\left(1-\rho^{2}\right)}(\right. \\
& \left(\frac{\log \left(e^{a x}-1\right)-\mu_{1}}{\sigma_{1}}\right)^{2}+\left(\frac{\log \left(e^{b y}-1\right)-\mu_{2}}{\sigma_{2}}\right)^{2}- \\
& \left.\left.\frac{2 \rho\left(\log \left(e^{a x}-1\right)-\mu_{1}\right)\left(\log \left(e^{b y}-1\right)-\mu_{2}\right)}{\sigma_{1} \sigma_{2}}\right)\right)
\end{aligned}
$$

where $x, y \geq 0, a, b, \sigma_{1}, \sigma_{2} \in \mathfrak{R}_{+}, \rho \in(-1,1), \mu_{1}, \mu_{2} \in \mathfrak{R}$.

Figure 2 shows the BNEE1 p.d.f. for $\mu_{1}=\mu_{2}=0, \sigma_{1}=\sigma_{2}=1, \rho=0$ and some values of $a$ and $b$. Figure 3 also shows the BNEE1 p.d.f. for $a=b=0.5$ and some variate values of $\mu_{1}, \mu_{2}, \sigma_{1}, \sigma_{2}$ and $\rho$.

Shannon entropy of the bivariate normal-X-Y type 1 distribution family using Theorem (3.1) will be obtained as

$$
\begin{aligned}
\eta_{X, Y}= & \eta_{U, S}+\mu_{U}+\mu_{V}+2 E_{U}\left(\log \left(1-\frac{e^{U}}{1+e^{U}}\right)\right)+2 E_{V}\left(\log \left(1-\frac{e^{V}}{1+e^{V}}\right)\right) \\
& -E_{U}\left(\log \left(f_{1}\left(F_{1}^{-1}\left(\frac{e^{U}}{1+e^{U}}\right)\right)\right)\right)-E_{V}\left(\log \left(f_{2}\left(F_{2}^{-1}\left(\frac{e^{V}}{1+e^{V}}\right)\right)\right)\right),
\end{aligned}
$$

where $\eta_{U, V}$ is the Shannon entropy of the bivariate normal distribution, $U$ and $V$ are the random variables with normal p.d.f. and $\mu_{U}$ and $\mu_{V}$ are the expectations of random variables $U$ and $V$, respectively. Estimates of the Shannon entropy of the bivariate normal p.d.f. have been obtained by Misra et al. (2005).

Let a random sample of size $n$ be taken from the bivariate density in (4.1), then the corresponding log-likelihood function can be written as

$$
\begin{aligned}
& l\left(\mu_{1}, \mu_{2}, \sigma_{1}, \sigma_{2}, \rho, a, b\right)=n \log a+n \log b-n \log 2 \pi-n \log \sigma_{1}-n \log \sigma_{2} \\
& -\sum_{i=1}^{n} \log \left(1-e^{-a x_{i}}\right)-\sum_{i=1}^{n} \log \left(1-e^{-b y_{i}}\right)-\frac{n}{2} \log \left(1-\rho^{2}\right) \\
& -\frac{1}{2\left(1-\rho^{2}\right)} \sum_{i=1}^{n}\left(\frac{\log \left(e^{a x_{i}}-1\right)-\mu_{1}}{\sigma_{1}}\right)^{2}-\frac{1}{2\left(1-\rho^{2}\right)} \sum_{i=1}^{n}\left(\frac{\log \left(e^{b y_{i}}-1\right)-\mu_{2}}{\sigma_{2}}\right)^{2} \\
& +\frac{\rho}{1-\rho^{2}} \sum_{i=1}^{n} \frac{\left(\log \left(e^{a x_{i}}-1\right)-\mu_{1}\right)}{\sigma_{1}} \frac{\left(\log \left(e^{b y_{i}}-1\right)-\mu_{2}\right)}{\sigma_{2}} .
\end{aligned}
$$


By differentiating (4.1) with respect to $\mu_{1}, \mu_{2}, \sigma_{1}, \sigma_{2}, \rho, a$ and $b$, we get

$$
\begin{aligned}
& \frac{\partial}{\partial \mu_{1}} l\left(\mu_{1}, \mu_{2}, \sigma_{1}, \sigma_{2}, \rho, a, b\right)=\frac{1}{\left(1-\rho^{2}\right) \sigma_{1}^{2}} \sum_{i=1}^{n}\left(\log \left(e^{a x_{i}}-1\right)-\mu_{1}\right) \\
& -\frac{\rho}{\sigma_{1} \sigma_{2}\left(1-\rho^{2}\right)} \sum_{i=1}^{n}\left(\log \left(e^{b y_{i}}-1\right)-\mu_{2}\right) \text {, } \\
& \frac{\partial}{\partial \mu_{2}} l\left(\mu_{1}, \mu_{2}, \sigma_{1}, \sigma_{2}, \rho, a, b\right)=\frac{1}{\left(1-\rho^{2}\right) \sigma_{2}^{2}} \sum_{i=1}^{n}\left(\log \left(e^{b y_{i}}-1\right)-\mu_{2}\right) \\
& -\frac{\rho}{\sigma_{1} \sigma_{2}\left(1-\rho^{2}\right)} \sum_{i=1}^{n}\left(\log \left(e^{a x_{i}}-1\right)-\mu_{1}\right) \\
& \frac{\partial}{\partial \sigma_{1}} l\left(\mu_{1}, \mu_{2}, \sigma_{1}, \sigma_{2}, \rho, a, b\right)=\frac{-n}{\sigma_{1}}+\frac{1}{\left(1-\rho^{2}\right) \sigma_{1}} \sum_{i=1}^{n}\left(\log \left(e^{a x_{i}}-1\right)-\mu_{1}\right)^{2} \\
& -\frac{\rho}{\sigma_{1}^{2} \sigma_{2}\left(1-\rho^{2}\right)} \sum_{i=1}^{n}\left(\log \left(e^{a x_{i}}-1\right)-\mu_{1}\right)\left(\log \left(e^{b y_{i}}-1\right)-\mu_{2}\right), \\
& \frac{\partial}{\partial \sigma_{2}} l\left(\mu_{1}, \mu_{2}, \sigma_{1}, \sigma_{2}, \rho, a, b\right)=\frac{-n}{\sigma_{2}}+\frac{1}{\left(1-\rho^{2}\right) \sigma_{2}} \sum_{i=1}^{n}\left(\log \left(e^{b y_{i}}-1\right)-\mu_{2}\right)^{2} \\
& -\frac{\rho}{\sigma_{2}^{2} \sigma_{1}\left(1-\rho^{2}\right)} \sum_{i=1}^{n}\left(\log \left(e^{b y_{i}}-1\right)-\mu_{2}\right)\left(\log \left(e^{a x_{i}}-1\right)-\mu_{1}\right), \\
& \frac{\partial}{\partial \rho} l\left(\mu_{1}, \mu_{2}, \sigma_{1}, \sigma_{2}, \rho, a, b\right)= \\
& \frac{n \rho}{1-\rho^{2}}-\frac{\rho}{\left(1-\rho^{2}\right)^{2}}\left(\sum_{i=1}^{n}\left(\frac{\log \left(e^{a x_{i}}-1\right)-\mu_{1}}{\sigma_{1}}\right)^{2}+\left(\frac{\log \left(e^{b y_{i}}-1\right)-\mu_{2}}{\sigma_{2}}\right)^{2}\right) \\
& +\frac{1+\rho^{2}}{\left(1-\rho^{2}\right)^{2}} \sum_{i=1}^{n} \frac{\left(\log \left(e^{a x_{i}}-1\right)-\mu_{1}\right)}{\sigma_{1}} \frac{\left(\log \left(e^{b y_{i}}-1\right)-\mu_{2}\right)}{\sigma_{2}}, \\
& \frac{\partial}{\partial a} l\left(\mu_{1}, \mu_{2}, \sigma_{1}, \sigma_{2}, \rho, a, b\right)= \\
& \frac{n}{a}-\sum_{i=1}^{n} \frac{x_{i} e^{-a x_{i}}}{1-e^{-a x_{i}}}-\frac{1}{\left(1-\rho^{2}\right) \sigma_{1}^{2}} \sum_{i=1}^{n}\left(\log \left(e^{a x_{i}}-1\right)-\mu_{1}\right) \frac{x_{i} e^{-a x_{i}}}{1-e^{-a x_{i}}} \\
& +\frac{\rho}{\sigma_{1} \sigma_{2}\left(1-\rho^{2}\right)} \sum_{i=1}^{n}\left(\log \left(e^{b y_{i}}-1\right)-\mu_{2}\right) \frac{x_{i} e^{-a x_{i}}}{1-e^{-a x_{i}}}
\end{aligned}
$$


and

$$
\begin{aligned}
& \frac{\partial}{\partial b} l\left(\mu_{1}, \mu_{2}, \sigma_{1}, \sigma_{2}, \rho, a, b\right)= \\
& \frac{n}{b}-\sum_{i=1}^{n} \frac{y_{i} e^{-b y_{i}}}{1-e^{-b y_{i}}}-\frac{1}{\left(1-\rho^{2}\right) \sigma_{2}^{2}} \sum_{i=1}^{n}\left(\log \left(e^{b y_{i}}-1\right)-\mu_{2}\right) \frac{y_{i} e^{-b y_{i}}}{1-e^{-b y_{i}}} \\
& +\frac{\rho}{\sigma_{1} \sigma_{2}\left(1-\rho^{2}\right)} \sum_{i=1}^{n}\left(\log \left(e^{a x_{i}}-1\right)-\mu_{1}\right) \frac{y_{i} e^{-b y_{i}}}{1-e^{-b y_{i}}} .
\end{aligned}
$$

Setting seven equations equal to zero and solving simultaneously, we get the maximum likelihood estimates for $\mu_{1}, \mu_{2}, \sigma_{1}, \sigma_{2}, \rho, a$ and $b$.

Suppose $a$ and $b$ are known, then according to Lemma 2.2 , the $\left(\log \left(e^{a X}-1\right), \log \left(e^{b Y}-1\right)\right)$ in (4.1) follows a bivariate normal p.d.f. with parameters $\mu_{1}, \mu_{2}, \sigma_{1}, \sigma_{2}$ and $\rho$. Then the estimates of $\mu_{1}, \mu_{2}, \sigma_{1}, \sigma_{2}$ and $\rho$ can be obtained as

$$
\begin{gathered}
\hat{\mu_{1}}=\frac{1}{n} \sum_{i=1}^{n} \log \left(e^{a x_{i}}-1\right), \\
\hat{\mu_{2}}=\frac{1}{n} \sum_{i=1}^{n} \log \left(e^{b y_{i}}-1\right), \\
\hat{\sigma_{1}}=\sqrt{\frac{1}{n} \sum_{i=1}^{n}\left(\log \left(e^{a x_{i}}-1\right)-\hat{\mu_{1}}\right)^{2}}=\sqrt{\frac{1}{n} \sum_{i=1}^{n} \log \frac{e^{a x_{i}}-1}{\left(\prod_{i=1}^{n}\left(e^{a x_{i}}-1\right)\right)^{\frac{1}{n}}}} \\
\hat{\sigma_{2}}=\sqrt{\frac{1}{n} \sum_{i=1}^{n}\left(\log \left(e^{b y_{i}}-1\right)-\hat{\mu_{2}}\right)^{2}}=\sqrt{\frac{1}{n} \sum_{i=1}^{n} \log \frac{e^{b y_{i}}-1}{\left(\prod_{i=1}^{n}\left(e^{b y_{i}}-1\right)\right)^{\frac{1}{n}}}}
\end{gathered}
$$

and

$$
\hat{\rho}=\frac{1}{n} \sum_{i=1}^{n} \frac{\left(\log \left(e^{a x_{i}}-1\right)-\hat{\mu_{1}}\right)\left(\log \left(e^{b y_{i}}-1\right)-\hat{\mu_{2}}\right)}{\hat{\sigma_{1}} \hat{\sigma_{2}}} .
$$

2. In Example 3.2, let $r(x, y), F_{1}(x)$ and $F_{2}(y)$ be the bivariate normal p.d.f., exponential c.d.f. with parameter $a$ and exponential c.d.f. with parameter $b$, respectively. Then the bivariate normal-exponential-exponential type 2 (BNEE2) p.d.f. will be obtained as

$$
\begin{aligned}
g(x, y) & =\frac{1}{x y} f_{\text {Bnorm }}(\log (a x), \log (b y)) \\
& =\frac{1}{2 \pi \sigma_{1} \sigma_{2} \sqrt{1-\rho^{2}} x y} \exp \left(-\frac{1}{2\left(1-\rho^{2}\right)}\left(\left(\frac{\log (a x)-\mu_{1}}{\sigma_{1}}\right)^{2}\right.\right. \\
& \left.+\left(\frac{\log (b y)-\mu_{2}}{\sigma_{2}}\right)^{2}-\frac{2 \rho\left(\log (a x)-\mu_{1}\right)\left(\log (b y)-\mu_{2}\right)}{\sigma_{1} \sigma_{2}}\right),
\end{aligned}
$$


where $x, y \geq 0, a, b, \sigma_{1}, \sigma_{2} \in \mathfrak{R}_{+}, \mu_{1}, \mu_{2} \in \mathfrak{R}$ and $\rho \in(-1,1)$.

This is the bivariate $\log$ normal p.d.f. with parameters $\mu_{1}-\log a, \mu_{2}-\log b, \sigma_{1}, \sigma_{2}$ and $\rho$ (see Cheng (1986)).

The marginal p.d.f. of the bivariate normal-X-Y family, $g_{X}(x)$ can be obtained from Theorem 2.3 as

$$
g_{X}(x)=f_{1}(x) W_{1}^{\prime}\left(F_{1}(x)\right) f_{\text {norm }}\left(W_{1}\left(F_{1}(x)\right)\right) .
$$

The obtained marginal p.d.f. follows the normal-X p.d.f. which can be obtained by the method of the $T-X$ family. Some properties of case 2 can be obtained similar to case 1 .

Analysis of these families of distributions needs a separate study but a simulated study of BNEE2 p.d.f. with different parameters has been done. Samples of size $n=200$ were produced for $m=300$ times. This p.d.f. has been determined with seven parameters $\mu_{1}, \mu_{2}, \sigma_{1}, \sigma_{2}, \rho, a$ and $b$ The bivariate random vector $(U, V)$ was simulated from the bivariate normal distribution and then $X=\frac{1}{a} e^{U}$ and $Y=\frac{1}{b} e^{V}$ were generated. The R software (mvnorm package) was used. The results have been shown in Table 1 . Table 1 shows the mean, variance, skewness, and kurtosis of $X$ and $Y$ and covariance of $(X, Y)$ for some values of $\mu_{1}, \mu_{2}, \sigma_{1}, \sigma_{2}, \rho, a$ and $b$. A glance at Table 1 shows that when $\mu_{1}$ and $\mu_{2}$ increase the means of $X$ and $Y$ increase. When $\rho$ increases, the covariance of $(X, Y)$ increases. When $a$ and $b$ increase the means of $X$ and $Y$ decrease. 

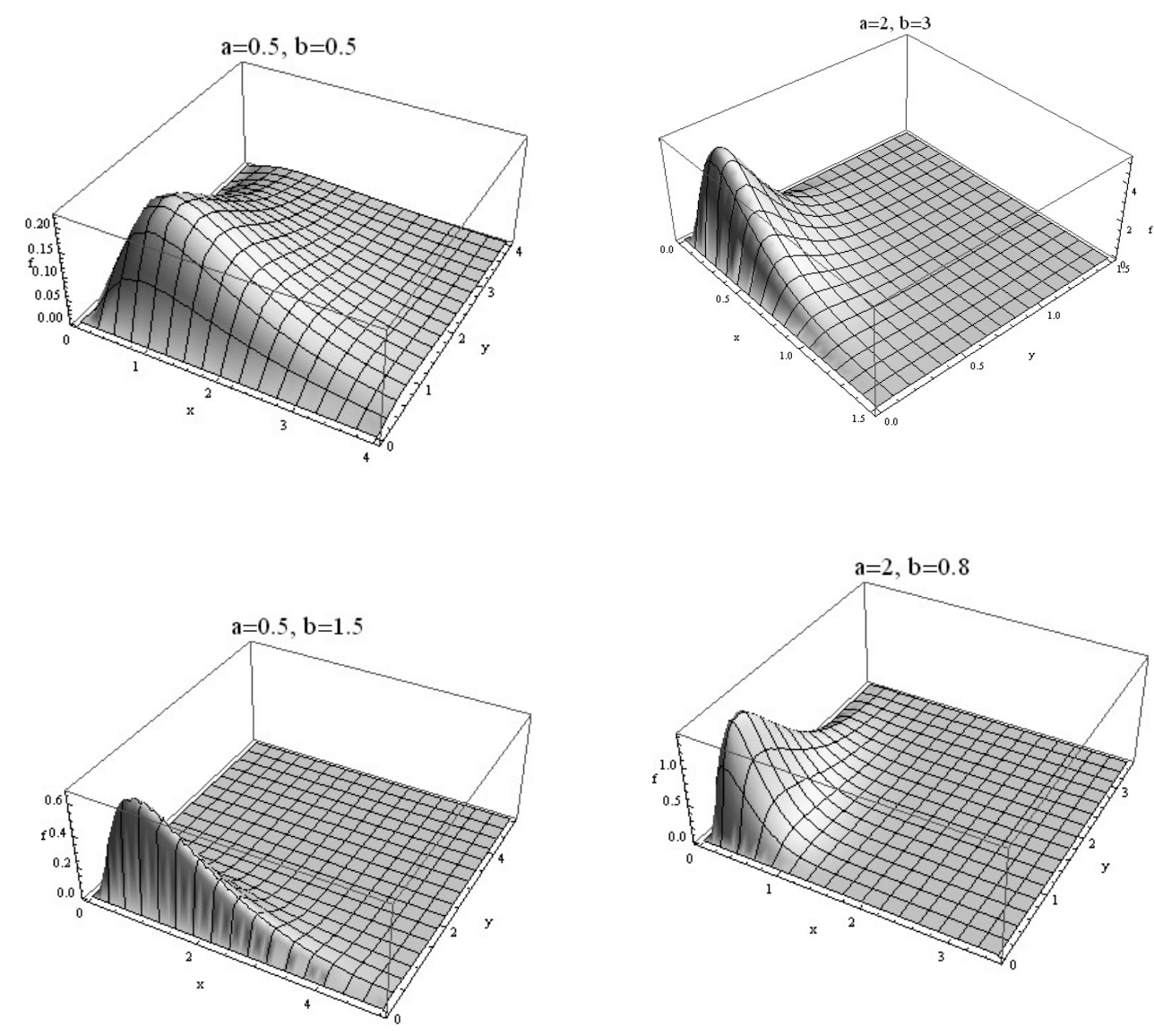

Figure 2: BNEE1 p.d.f. with parameters $\mu_{1}=\mu_{2}=0, \sigma_{1}=\sigma_{2}=1, \rho=0$ and for some values of $a$ and $b$ 

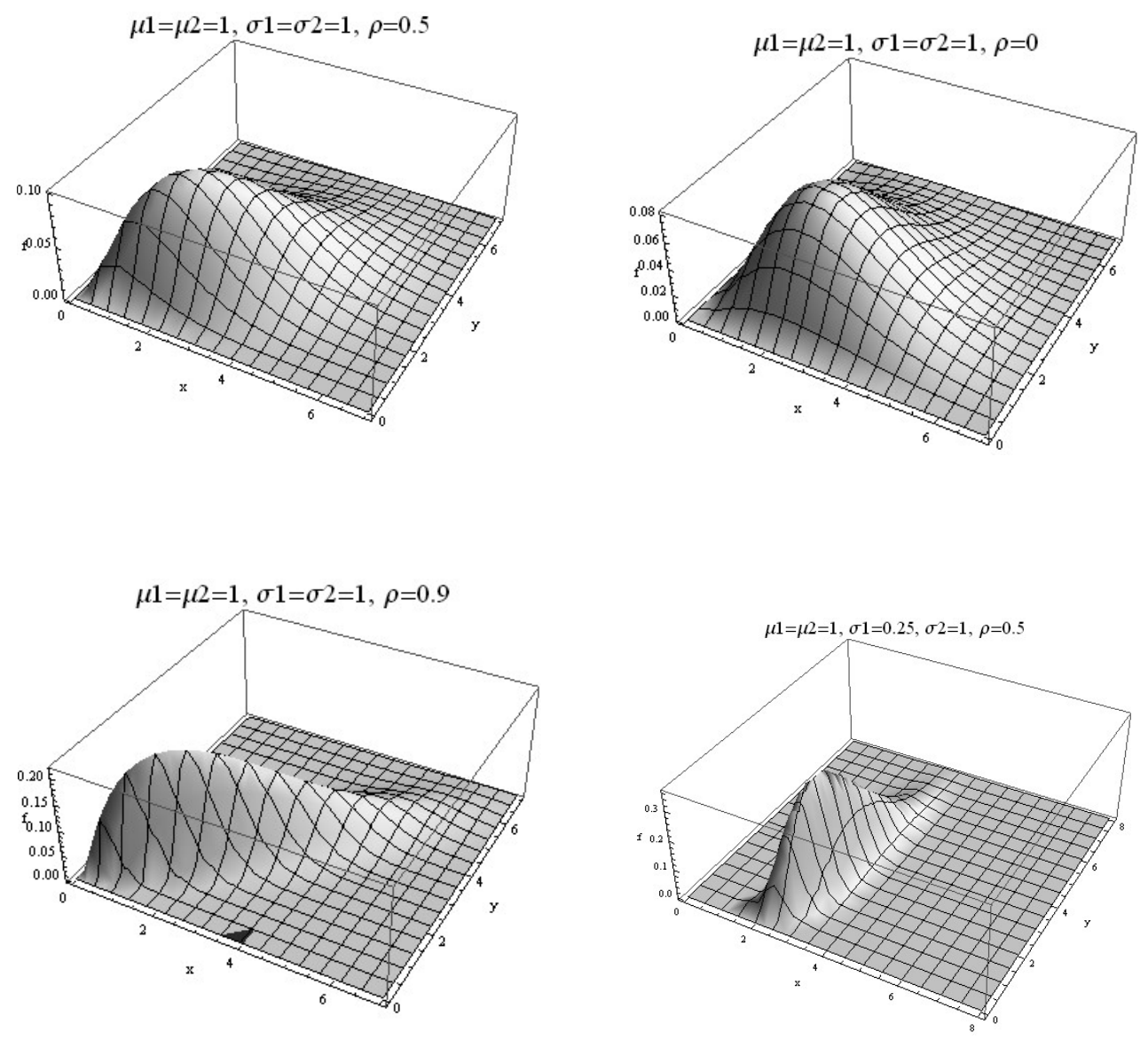

Figure 3: BNEE1 p.d.f. with parameters $a=b=0.5$ and for some values of $\mu_{1}, \mu_{2}, \sigma_{1}, \sigma_{2}$ and $\rho$ 


\subsection{Bivariate Gamma-X-Y Distribution Family}

Mckay (1934) introduced a kind of bivariate gamma distribution with p.d.f.

$$
f_{B G}(u, v)=\frac{c^{a+b}}{\Gamma[a] \Gamma[b]} u^{a-1}(v-u)^{b-1} e^{-c v}, \quad a, b, c>0, \quad v>u>0 .
$$

The marginal distributions of $X$ and $Y$ are gamma with shape parameters $a$ and $a+b$, respectively, but they have a common scale parameter $\frac{1}{c}$.

1. In (2.2), let $r(x, y)$ be the bivariate gamma p.d.f. Also, let $W_{1}\left(F_{1}(x)\right)=\frac{F_{1}(x)}{1-F_{1}(x)}$ and $W_{2}\left(F_{2}(y)\right)=$ $\frac{F_{2}(y)}{1-F_{2}(y)}$, then the bivariate gamma-X-Y p.d.f. is obtained for $F_{1}(x) \leq F_{2}(y)$ as

$$
\begin{aligned}
g(x, y)= & \frac{f_{1}(x) f_{2}(y)}{\left(1-F_{1}(x)\right)^{2}\left(1-F_{2}(y)\right)^{2}} f_{B G}\left(\frac{F_{1}(x)}{1-F_{1}(x)}, \frac{F_{2}(y)}{1-F_{2}(y)}\right) \\
= & \frac{c^{a+b} e^{-\frac{c F_{2}(y)}{1-F_{2}(y)}} f_{1}(x) f_{2}(y)}{\Gamma[a] \Gamma[b]\left(1-F_{1}(x)\right)^{2}\left(1-F_{2}(y)\right)^{2}}\left(\frac{F_{1}(x)}{1-F_{1}(x)}\right)^{a-1} \times \\
& \left(\frac{F_{2}(y)}{1-F_{2}(y)}-\frac{F_{1}(x)}{1-F_{1}(x)}\right)^{b-1} .
\end{aligned}
$$

Now, let $F_{1}$ and $F_{2}$ be the normal density functions, then the bivariate gamma-normalnormal type 1 distribution family (BGNN1) will be obtained. The marginal density functions of $X$ and $Y$ can be, respectively, obtained from Theorem 2.3 as

$$
g_{X}(x)=\frac{c^{a} f_{1}(x)}{\Gamma[a]\left(1-F_{1}(x)\right)^{2}}\left(\frac{F_{1}(x)}{1-F_{1}(x)}\right)^{a-1} e^{-\frac{c F_{1}(x)}{\left(1-F_{1}(x)\right)}}
$$

and

$$
g_{Y}(y)=\frac{c^{a+b} f_{2}(y)}{\Gamma[a+b]\left(1-F_{2}(y)\right)^{2}}\left(\frac{F_{2}(y)}{1-F_{2}(y)}\right)^{a+b-1} e^{-\frac{c F_{2}(y)}{\left(1-F_{2}(y)\right)}} .
$$

2. Let, in (2.2), $r(x, y)$ be the bivariate gamma p.d.f. Also let $W_{1}\left(F_{1}(x)\right)=-\log \left(1-F_{1}(x)\right)$ and $W_{2}\left(F_{2}(y)\right)=-\log \left(1-F_{2}(y)\right)$. Then

$$
\begin{aligned}
g(x, y)= & \frac{f_{1}(x) f_{2}(y)}{\left(1-F_{1}(x)\right)\left(1-F_{2}(y)\right)} f_{B G}\left(-\log \left(1-F_{1}(x)\right),-\log \left(1-F_{2}(y)\right)\right) \\
= & \frac{c^{a+b} f_{1}(x) f_{2}(y)}{\Gamma[a] \Gamma[b]\left(1-F_{1}(x)\right)\left(1-F_{2}(y)\right)} e^{c \log \left(1-F_{2}(y)\right)} \\
& \times\left(-\log \left(1-F_{1}(x)\right)\right)^{a-1}\left(-\log \left(1-F_{2}(y)\right)+\log \left(1-F_{1}(x)\right)\right)^{b-1}
\end{aligned}
$$




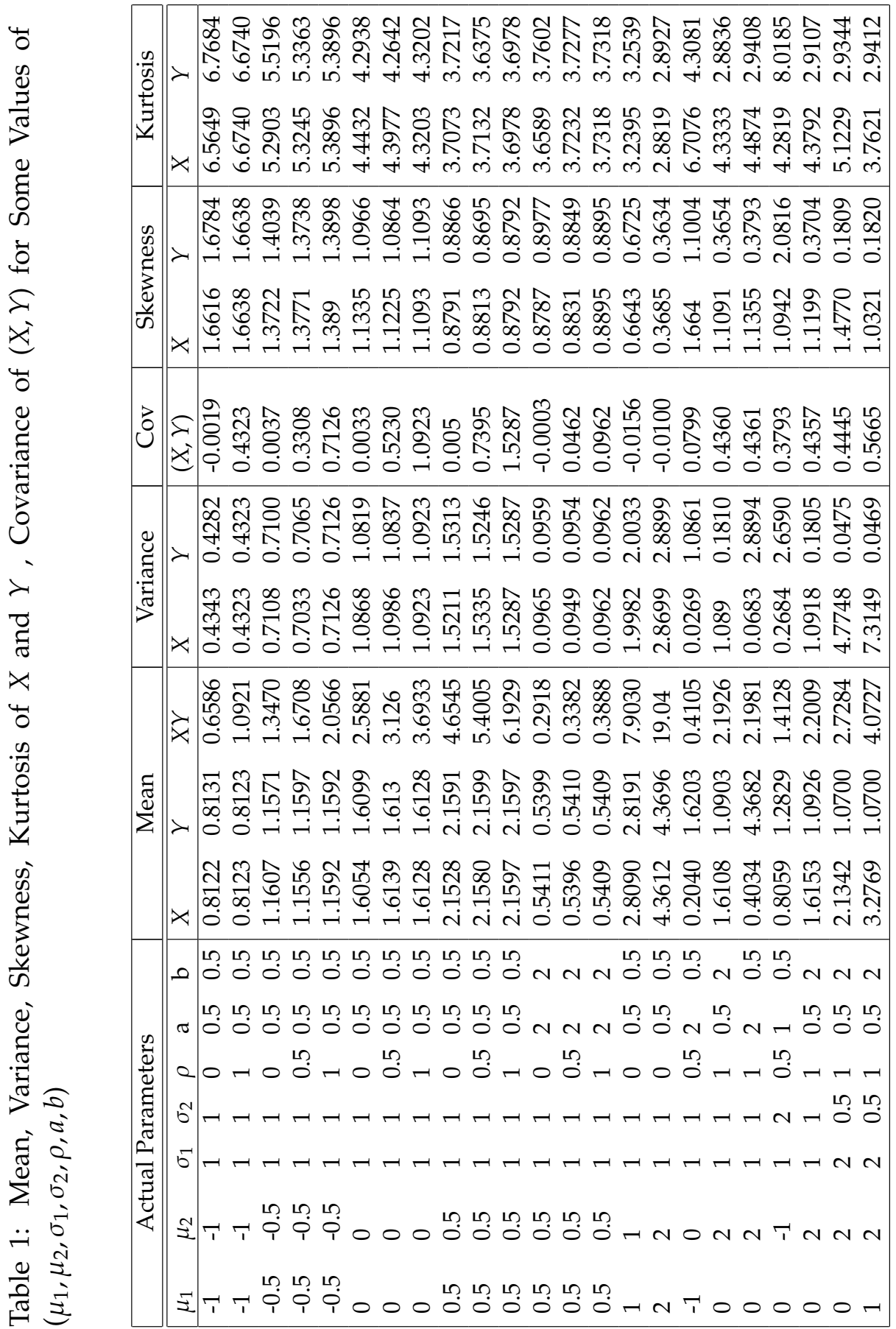


The marginal density functions of $X$ and $Y$ can be obtained as

$$
g_{X}(x)=\frac{c^{a} f_{1}(x)}{\Gamma[a]}\left(-\log \left(1-F_{1}(x)\right)\right)^{a-1}\left(1-F_{1}(x)\right)^{c-1}
$$

and

$$
g_{Y}(y)=\frac{c^{a+b} f_{2}(y)}{\Gamma[a+b]}\left(-\log \left(1-F_{2}(y)\right)\right)^{a+b-1}\left(1-F_{2}(y)\right)^{c-1} .
$$

The marginals follow the gamma-X family that have been introduced by Alzaatreh et al. (2014).

\subsection{Bivariate Weibull-X-Y Distribution Family}

The bivariate Weibull p.d.f. is defined for $0<\alpha \leq 1, \xi_{1}, \xi_{2}, k>0, \phi=\frac{1}{\alpha}$ and $t, s \geq 0$ as follows:

$$
\begin{aligned}
f_{B W}(t, s) & =k^{2} \xi_{1}^{\phi} \xi_{2}^{\phi} t^{k \phi-1} s^{k \phi-1} \\
& \times\left\{\left(\xi_{1}^{\phi} t^{k \phi}+\xi_{2}^{\phi} s^{k \phi}\right)^{2 \alpha-2}+(\phi-1)\left(\xi_{1}^{\phi} t^{k \phi}+\xi_{2}^{\phi} s^{k \phi}\right)^{\alpha-2}\right\} \\
& \times \exp \left(-\left(\xi_{1}^{\phi} t^{k \phi}+\xi_{2}^{\phi} s^{k \phi}\right)^{\alpha}\right) .
\end{aligned}
$$

The marginal distributions are Weibull with parameters $\frac{1}{\xi}$ and $k$. For more information the reader can refer to Kotz et al. (2000).

1. Let, in (2.2), $r(x, y)$ be the bivariate Weibull p.d.f. with $0<\alpha \leq 1, k>0$. Suppose $W_{1}\left(F_{1}(x)\right)=\frac{F_{1}(x)}{1-F_{1}(x)}$ and $W_{2}\left(F_{2}(y)\right)=\frac{F_{2}(y)}{1-F_{2}(y)}$, then the bivariate Weibull-X-Y p.d.f. is given as

$$
g(x, y)=\frac{f_{1}(x) f_{2}(y)}{\left(1-F_{1}(x)\right)^{2}\left(1-F_{2}(y)\right)^{2}} f_{B W}\left(\frac{F_{1}(x)}{1-F_{1}(x)}, \frac{F_{2}(y)}{\left.1-F_{2}(y)\right)}\right) .
$$

Then

$$
\begin{aligned}
g(x, y) & =\frac{k^{2} \xi_{1}^{\phi} \xi_{2}^{\phi} f_{1}(x) f_{2}(y)}{\left(1-F_{1}(x)\right)^{2}\left(1-F_{2}(y)\right)^{2}}\left(\frac{F_{1}(x)}{1-F_{1}(x)}\right)^{k \phi-1}\left(\frac{F_{2}(y)}{1-F_{2}(y)}\right)^{k \phi-1} \\
& \times\left\{\left(\xi_{1}^{\phi}\left(\frac{F_{1}(x)}{1-F_{1}(x)}\right)^{k \phi}+\xi_{2}^{\phi}\left(\frac{F_{2}(y)}{1-F_{2}(y)}\right)^{k \phi}\right)^{2 \alpha-2}\right. \\
& \left.+(\phi-1)\left(\xi_{1}^{\phi}\left(\frac{F_{1}(x)}{1-F_{1}(x)}\right)^{k \phi}+\xi_{2}^{\phi}\left(\frac{F_{2}(y)}{1-F_{2}(y)}\right)^{k \phi}\right)^{\alpha-2}\right\} \\
& \times \exp \left(-\left(\xi_{1}^{\phi}\left(\frac{F_{1}(x)}{1-F_{1}(x)}\right)^{k \phi}+\xi_{2}^{\phi}\left(\frac{F_{2}(y)}{1-F_{2}(y)}\right)^{k \phi}\right)^{\alpha}\right) .
\end{aligned}
$$


2. Let $r, F_{1}(x)$ and $F_{2}(y)$ be the bivariate Weibull, Rayleigh with parameter $b_{1}$ and Rayleigh with parameter $b_{2}$, respectively. Also, in $(2.2)$, let $W_{1}\left(F_{1}(x)\right)=-\log \left(1-F_{1}(x)\right)$ and $W_{2}\left(F_{2}(y)\right)=$ $-\log \left(1-F_{2}(y)\right)$, then the bivariate Weibull-Rayleigh-Rayleigh type 2 (BWRR2) p.d.f. will be obtained as follows:

$$
\begin{aligned}
g(x, y) & =\frac{k^{2} \xi_{1}^{\phi} \xi_{2}^{\phi}}{\left(b_{1} b_{2}\right)^{2}} x y\left(\frac{x}{\sqrt{2} b_{1}}\right)^{2 k \phi-2}\left(\frac{y}{\sqrt{2} b_{2}}\right)^{2 k \phi-2} \times\left\{\left(\xi_{1}^{\phi}\left(\frac{x}{\sqrt{2} b_{1}}\right)^{2^{k \phi}}+\xi_{2}^{\phi}\left(\frac{y}{\sqrt{2} b_{2}}\right)^{2^{k \phi}}\right)^{2 \alpha-2}\right. \\
& +(\phi-1)\left(\xi_{1}^{\phi}\left(\frac{x}{\sqrt{2} b_{1}}\right)^{2^{k \phi}}+\xi_{2}^{\phi}\left(\frac{y}{\sqrt{2} b_{2}}\right)^{2^{k \phi}}\right)^{\alpha-2} \\
& \times \exp \left(-\left(\xi_{1}^{\phi}\left(\frac{x}{\sqrt{2} b_{1}}\right)^{2^{k \phi}}+\xi_{2}^{\phi}\left(\frac{y}{\sqrt{2} b_{2}}\right)^{\left.2^{k \phi}\right)^{\alpha}}\right)\right\}
\end{aligned}
$$

where $0<\alpha \leq 1, k, b_{1}, b_{2}>0, \phi=\frac{1}{\alpha}$ and $x, y \geq 0$. The marginal p.d.f. of $X$ will be as:

$$
g_{X}(x)=\frac{x \xi_{1} k}{b_{1}^{2}}\left(\frac{\xi_{1} x^{2}}{2 b_{1}^{2}}\right)^{k-1} \exp \left(-\xi_{1} \frac{x^{2}}{2 b_{1}^{2}}\right)^{k}, x>0 .
$$

This p.d.f. is the Weibull-Rayleigh p.d.f. that has been introduced by Ganji et al. (2016).

\section{Application}

In this section, the BWRR2 in (4.3) is applied to a real data set. The data set represents the scores of 25 first year graduate students in Probability-I and Inference-I in a premier Institute in India. This data set has been analyzed by Al-Mutairi et al. (2011). The data are

$$
\begin{aligned}
X= & (53,55,85,87,22,23,25,93,51,62,53,32,43,47,30,88,59,49,42, \\
& 71,41,82,75,93,37), \\
Y= & (89,90,59,50,25,29,54,62,39,25,89,32,33,63,38,77,55,41,31, \\
& 66,57,32,43,88,34) .
\end{aligned}
$$

They fitted these data to the marginals of Bivariate distribution with Weighted Exponential marginals (BWE). They used Kolmogrov-Smirnov distance and corresponding p-values between the empirical distribution function of the marginals. We fit the marginals of BWRR2 to these data. Kolmogrov-Smirnov test statistics (K-S), $p$-value for the fitted distributions, Akaike Information criterion (AIC) and Bayesian information criterion (BIC) are reported in Table 2. The results in Table 2 show that the marginals of the BWRR2 are much better than BWE. The maximum likelihood estimates of the parameters of the marginals of $X$ and $Y$ are $(20.5554,1.3770,9.8349)$ and $(20.1717,1.3408,9.2528)$, respectively. 
Table 2: The Kolmogrov-Smirnov distance and corresponding p-values, AIC and BIC for the scores data

\begin{tabular}{|c|c|c|c|c|c|}
\hline Distribution & Variable & $\mathrm{K}-\mathrm{S}$ & $\mathrm{p}$-value & $\mathrm{AIC}$ & $\mathrm{BIC}$ \\
\hline BWE & $X$ & 0.208 & 0.329 & 241.6494 & 244.0872 \\
& $Y$ & 0.225 & 0.286 & 237.5752 & 240.0130 \\
\hline BWRR2 & $X$ & 0.1127 & 0.9086 & 230.7994 & 234.4560 \\
& $Y$ & 0.1288 & 0.8012 & 227.4621 & 231.1187 \\
\hline
\end{tabular}

\section{Conclusion}

This study introduces a new generating method for bivariate distributions. This technique is a generalization of the $T-X$ univariate distribution family. In addition to the afore-mentioned examples, this technique can be used to examine the bivariate Gumbel-X-Y distribution, bivariate Pareto-X-Y and many other distributions whose properties and usages can be examined. Lastly, we discuss the application of a special distribution of this family. This method can be generalized to multivariate distributions. The aim of this study was merely to introduce a new method and to present several examples. Separate studies are required to describe more properties and applications.

\section{References}

Al-Mutairi, K., Ghitany, M. E. and Kundu, D. (2011), A new Bivaraite distribution with weighted exponential marginals and its multivariate generalization, Statistical papers, 52, 921-936.

Alzaatreh, A., Famoye, F. and Lee, C. (2012), Gamma-Pareto distribution and its applications. Journal of Modern Applied Statistical Methods , 11(1) , 78-94.

Alzaatreh, A., Lee, C. and Famoye, F. (2013, a), A new method for generating families of continuous distributions. Metron, 71, 63-79.

Alzaatreh, A., Famoye, F. and Lee, C. (2013, b), Weibull-Pareto distribution and its applications. Communications in Statistics:Theory \& Methods ,42(9) , 1673-1691.

Alzaatreh, A., Famoye, F. and Lee, C. (2014), The gamma-normal distribution: Properties and applications, Computational Statistics \& Data Analysis, 69 ,67-80.

Alzaatreh, A. and Knight, K. (2013), On The Gamma-Half Normal Distribution and Its Applications. Journal of Modern Applied Statistical Methods , 12(1),103-119.

Arnold, B. C., Castillo, E. and Sarabia, J. M. (1999). Conditional Specification of Statistical Models. Springer Series in Statistics. Springer, Heidelberg, New York. 
Cheng, Y. S. (1986), Bivariate log normal distribution for characterizing asbestos fiber aerosols, Aerosols Science and technology, 5(3), 359-368.

Cover, T. and Thomas, J. (2006), Elements of information theory, New Jersey: Wiley.

Ganji, M., Bevrani, H., Hami Golzar, N. and Zabihi. S. (2016), The Weibull-Rayleigh distribution, some properties and application, Journal of Mathematical Sciences, 3, 269-277.

Gray, R. (1990), Entropy and information theory, New York: Springer.

Hutchinson, T. P. and Lai, C. D. (1990). Continuous Bivariate Distributions, Emphasizing Applications, Rumsby Scientific Press, Adelaide, Australia.

Joe, H. (1997). Multivariate Models and Dependence Concepts. Chapman and Hall, New York.

Kotz, S., Balakrishnan, N. and Johnson, N. (2000), Continuous Multivariate Distributions. New York: Wiley.

Mckay, A. T. (1934), Sampling from batches, Journal of royal statistical society, Supplement, 1, 207-216.

Misra, N. Singh, H. Demchuk, E. (2005), Estimation of the entropy of the multivariate normal distribution, Journal of Multivariate Analysis, 92, 324-342.

Nelsen, R. B. (2006). An Introduction to Copulas. Second Edition. Springer, New York. arbitrary phase, Philosophical Magazine, 10, 73-78.

Shannon, C. E. (1948), A mathematical theory of communication, Bell System Technical Journal, $27,379-432$.

Torabi H. and Montazeri, N. H. (2012), The gamma-uniform distribution and its applications, Kybernetika, 48, 16-30.

Torabi, H. and Montazeri, N. H. (2014), The logistic-uniform distribution and its application, Communication in Statistics- Theory and Methods, 43(10), 2551-2569 